\title{
Is Continent Urinary Diversion Feasible in Children under Five Years of Age?
}

\author{
Luiz L. Barbosa, Riberto Liguori, Sergio L. Ottoni, Ubirajara Barroso Jr, Valdemar Ortiz, \\ Antonio Macedo Junior
}

Division of Urology, Federal University of Sao Paulo, Sao Paulo, Brazil

\begin{abstract}
Purpose: To review our clinical experience with urinary continent catheterizable reservoir in children under five years of age.

Materials and Methods: A total of 23 patients (16 males, 7 females) with a median age of 3.64 years were evaluated. Among these, 6 (26.08\%) had a posterior urethral valve, 9 (39.13\%) myelomeningocele, 4 (17.39\%) bladder exstrophy, 2 $(8.69 \%)$ genitourinary rabdomyosarcoma, $1(4.34 \%)$ had spinal tumor and $1(4.34 \%)$ an ano-rectal anomaly.

Results: Perioperative complications were observed in four patients consisting of one febrile urinary tract infection, one partial operative wound dehiscence, one partial stomal dehiscence and one vesico-cutaneous fistula after a secondary exstrophy repair. The overall long-term complications rate was $40.90 \%$ and consisted of two stomal stenoses $(9.09 \%)$, one neobladder mucosal extrusion (4.54\%), three neobladder calculi (13.63\%) and persistence of urinary incontinence in three patients $(13.63 \%)$. The overall surgical revision was $36.36 \%$ and final continence rate was $95.45 \%$ with mean follow-up of 39.95 months

Conclusion: Continent urinary diversion is technically feasible even in small children, with acceptable rates of complications.
\end{abstract}

Key words: children; congenital anomalies; urinary diversion; continence

Int Braz J Urol. 2009; 35: 459-66

\section{INTRODUCTION}

The ability to void spontaneously may be compromised in children with various congenital urologic abnormalities (neurogenic bladder, posterior urethral valve and bladder exstrophy). Although clean intermittent catheterization (CIC), pharmacological treatment and overnight catheter drainage has changed the natural history of most of these uropathies., it is not always possible to avoid renal deterioration in these patients owing to a combination of high detrusor pressure, infection and vesicoureteral reflux, moreover, urethral catheterization can be painful or not possible in female patients bound to a wheel chair.
The use of continent urinary reservoirs has gained wide acceptance, because when this procedure is combined with CIC, patients are often able to achieve continence with low intravesical pressure. If necessary, a continent catheterizable urinary reservoir and a concomitant bladder neck procedure can be helpful to restore continence.

On the other hand, each type of augmentation cystoplasty involves short-term and long-term morbidity (bacteriuria, pyelonephritis, mucus production, stone disease, metabolic disturbances, bladder rupture/perforation and malignancy) that may lead to impaired linear growth in young children $(1,2)$. To our knowledge, there is no consensus in the literature as 
regards patients' initial age when this procedure can be safely performed. The majority of the series report bladder augmentation in patients above 5 years of age $(>5$ years).

We retrospectively present our experience with orthotopic continent urinary diversion in children under five years of age. We evaluated all continent catheterizable procedures that were performed in our institution, to investigate if continent urinary diversion is technically feasible and safe even in younger children.

\section{MATERIALS AND METHODS}

The records of children who underwent a Macedo's continent catheterizable reservoir below five years of age were retrospectively reviewed. A total of 23 patients ( 16 males, 7 females) met the inclusion criteria in our present series of 115 procedures. Patient age at surgery varied from 1 to 5 years with median age of 3.64 years. All children had previously undergone urodynamic evaluation, except those with rabdomyosarcoma and bladder exstrophy.

The technique consists of a continent catheterizable ileum-based reservoir (3). A $30 \mathrm{~cm}$ segment of the distal ileum is isolated and bowel continuity restored by end-to-end anastomosis. The detubularization of the ileal segment follows the antimesenteric border of the intestine up to the middle of the segment. Here the incision line continues transversally to the anterior surface of the ileum, reaching the mesenteric border. A $3 \mathrm{~cm}$ horizontal incision along the mesenteric side is continued before returning to its usual direction at the antimesenteric border. The remainder of the ileum is then opened longitudinally (Figure-1A). The $3 \mathrm{~cm}$ flap from the anterior surface of the middle part of the ileum is mobilized cranially, and tubularized around a 12F silicone Foley catheter (Figure-1B). The continence valve mechanism is produced by embedding the tube over a serous-lined extramural tunnel created by polypropylene 3/0 sutures (Figure-1C). The distal end of the tube is anastomosed into a V-shaped to the skin flap to avoid stomal stenosis. The reservoir is anastomosed to the bladder or used as a substitute.

Surgical indications included patients with low bladder compliance and capacity, filling detrusor pressure above $40 \mathrm{~cm} \mathrm{H} 2 \mathrm{O}$, and/or urinary incontinence that had failed to conservative management (CIC and anticolinergics).

Among the patients, $6(26.08 \%)$ had posterior urethral valve (PUV), 9 (39.13\%) myelomeningocele, $4(17.39 \%)$ bladder exstrophy, 2 (8.69\%) genitourinary rabdomyosarcoma, $1(4.34 \%)$ spinal tumor and $1(4.34 \%)$ had an ano-rectal anomaly (Table-1). Four patients $(17.39 \%)$ had undergone either a vesicostomy or nephrostomy prior to bladder augmentation. One patient had bladder augmentation 14 months before kidney transplantation (Table-1).

The procedure was performed with the ileum in 21 patients $(91.30 \%)$ and transverse colon in 2 patients $(8.69 \%)$. The colon was used in two cases of genitourinary rhabdomyosarcoma, due to the risk of using a previous irradiated ileum to perform bladder substitution (4). In only these two patients, the technique was used as a pouch whereas in the others it was used as a bladder augmentation.

In one patient (4.34\%), an antegrade left colon enema procedure was performed in combination with bladder augmentation due to fecal incontinence (5).

Two of the 21 patients $(8.69 \%)$ underwent a bladder neck procedure, due to persistent leakage and low detrusor leak point pressure (6).

The patients' medical records were reviewed in order to define complication rates (acute and chronic), surgical revision rate and final continence.

\section{RESULTS}

A perioperative complication was observed in four patients consisting of one febrile urinary tract infection, one partial operative wound dehiscence, one partial stomal dehiscence and one vesico-cutaneous fistula after a secondary exstrophy repair. This last patient, developed sepsis due to fungal infection and did not survive.

The overall long-term complications rate was $40.90 \%$ and consisted of two stomal stenoses (9.09\%), one neobladder mucosal extrusion (4.54\%), three neobladder calculi (13.63\%) and persistence of urinary incontinence in three cases $(13.63 \%)$, Table-2. 
$1 \mathrm{~A}$
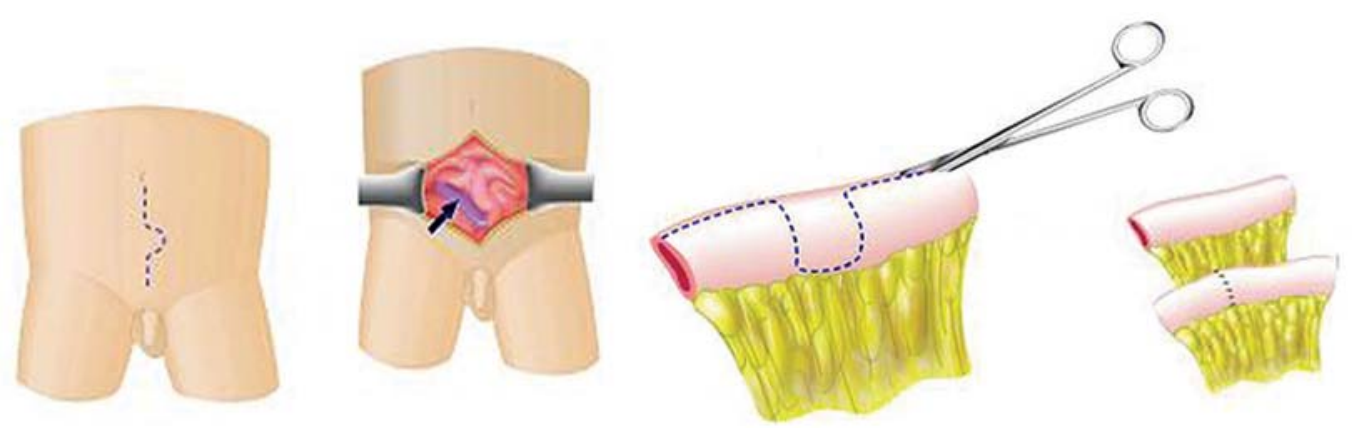

1B
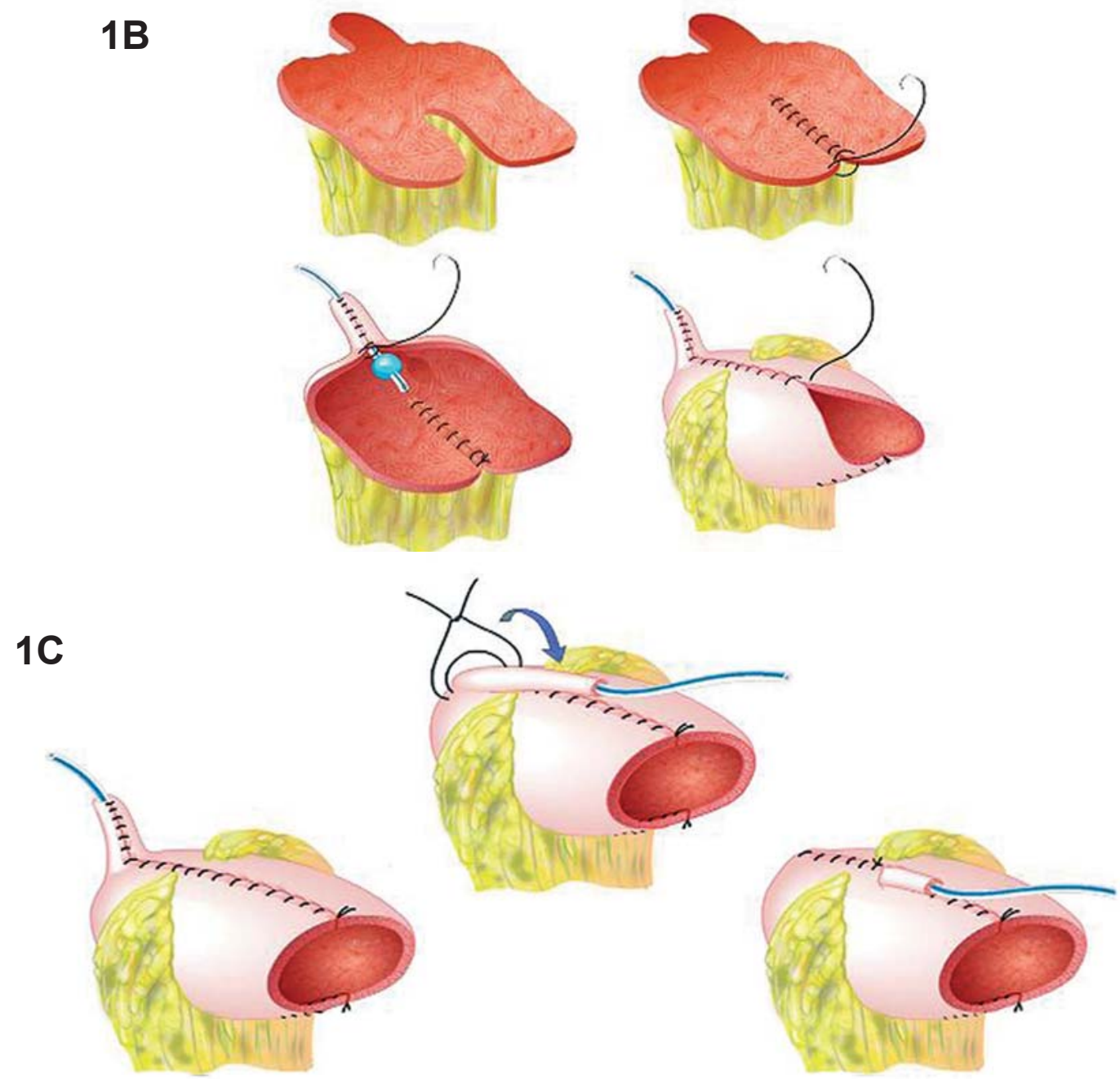

Figure 1 - Macedo's continent catheterizable reservoir - surgical technique. Reproduced from Macedo A. Jr., Srougi M.: A continent catheterizable ileum-based reservoir. BJU Int. 2000; 85: 160-2. 


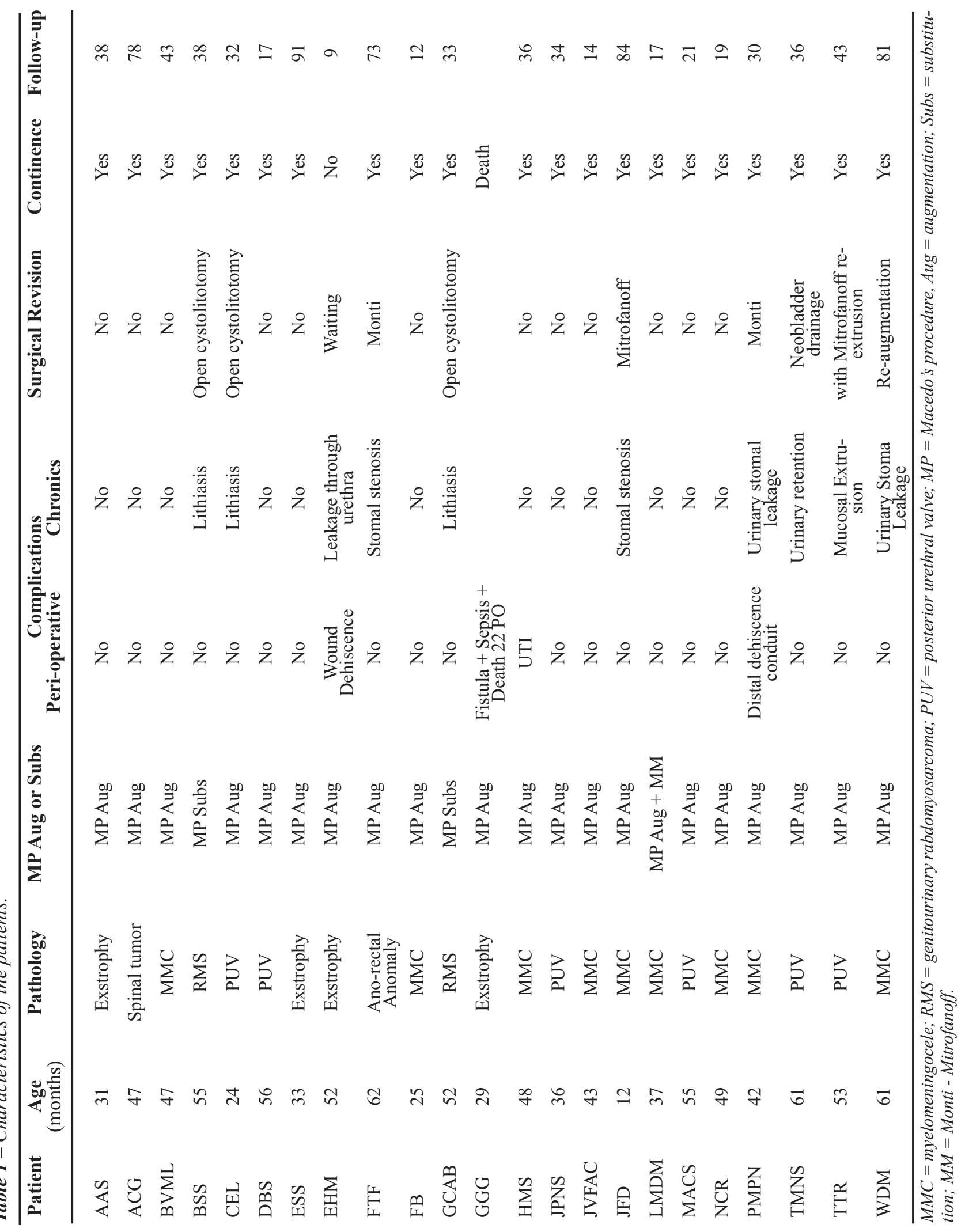


Table 2 - Overall complication rate, surgical revision and final continence.

\begin{tabular}{lc}
\hline General Complication Rate & $\begin{array}{c}\text { Absolute Number/ \% } \\
\text { Acute }\end{array}$ \\
Chronic & $9 / 17.39 \%$ \\
Chronic Complications & \\
Stomal stenosis & $2 / 21.73 \%$ \\
Lithiasis & $3 / 13.63 \%$ \\
Persistent leakage & $3 / 13.63 \%$ \\
Urethral & $2 / 9.09 \%$ \\
Stomal & $1 / 4.54 \%$ \\
Neobladder extrusion & $1 / 4.54 \%$ \\
Surgical Revision & $8 / 36.36 \%$ \\
Final Continence & $21 / 95.45 \%$ \\
\hline
\end{tabular}

In the two cases of stomal stenosis, surgical revision was necessary in both cases because progressive stomal dilatation failed.

The patient with neobladder mucosal extrusion $(4.54 \%)$ underwent redo closure and apendicovesicostomy.

Three patients presented with persistence of urinary leakage (13.63\%), two leaking through the stoma and one patient through the urethra after failed bladder neck surgery. The patients with stomal urinary leakage $(9.09 \%)$ were reoperated: one patient underwent a Monti procedure and the other a re-augmentation and they are currently continent.

Three patients developed reservoir calculi (13.63\%), and underwent open cystolitotomy after failed endoscopic attempt. All cases were found to have the calculi grown on a suture line presumably associated to one of the seromuscular Prolene sutures of the tube valvular mechanism.

One patient presented with late urinary retention due to overdistention of the reservoir and tube outlet, and a cystoscopy was necessary to help catheter passage through the stoma.

The overall surgical revision was $36.36 \%$ and final continence rate was $95.45 \%$ with mean followup of 39.95 (9 to 91) months. The only incontinent patient was a complex exstrophy form that failed a bladder neck procedure and is currently awaiting surgery (Table-1).

\section{COMMENTS}

Successful continent abdominal diversion requires a low pressure reservoir, a continent efferent channel and a good cosmetic and non stenotic skin stoma.

The choice of the intestinal segment to construct the reservoir is made according to surgeons' experience, but factors like incidence of electrolyte reabsorption and loss (acidosis and alcalosis) certainly play a role in this procedure.

The creation of a continent catheterizable conduit was initially described by Mitrofanoff, using the appendix as a catheterizable stoma. Although the appendix is the most popular channel, numerous other options have been reported (ureter, fallopian tubes and tubularized colonic or bladder flaps) (7). In cases when the appendix is absent, too short or has evidence of luminal stenosis, the search for an alternative is imperative. The Yang-Monti procedure consists of using a small segment of bowel (usually ileum) to create an efferent tube. A 2.0-2.5 cm segment of bowel is detubularized in the antimesenteric border resulting in a tube of 6-7 cm in length, when transversely retubularized. To date, the Yang-Monti technique is considered the substitute of choice of the appendix for the Mitrofanoff principle (8).

In Mitrofanoff and Yang-Monti procedures, when concomitant bladder augmentation or substitution is needed, two segments of bowel must be prepared for creation of a neobladder and an efferent tube. After that, both structures have to be joined together. The Macedo's continent catheterizable reservoir allows both procedures using the same segment of the bowel. We recently performed an experimental study to define the efficacy of the technique, in addition to other factors that may influence continence of catheterizable channels using the principle of embedding the outlet tube. From 20 pigs, colon specimens with $25 \mathrm{~cm}$ length were obtained and a transverse flap in the average point of the intestine was incised to create an efferent tube. A pressure study of both 
intra-luminal surface and channel was then conducted during the filling, of the submersed piece with environmental air in a water container, to define the efferent channel continence. This study showed that angulation of channel with colon, maintained by only one stitch was more important than a larger extension of the valve, represented by 3 sutures in order to allow continence to the efferent channel. This experimental study reproduced the clinical situation in our patients and outlined the need of anchoring the reservoir to the abdominal wall to retain the angulation of the stoma (9).

Leslie et al. (8) recently reviewed their series of Monti urinary channels performed from 1997 to 2004. These authors identified 168 patients with age of 10 months to 31 years at surgery. Mean follow-up was 2, 6 years. In 168 patients, the ileum was used to create the channel in $165(98.2 \%)$, while sigmoid colon was used in the other 3. A single Monti channel was created in $99(58.9 \%)$ and a spiral Monti in 66 $(39.3 \%)$. A total of 37 open revisions were required (18.5\%).Of these 31 patients, 26 required only 1 open revision, 4 required 2 revisions, and 1 patient required 3 revisions. Of the 152 patients, 148 were completely continent (148 of 152 or 97, 4\%), 1 had rare leakage, 2 had occasional or intermittent leakage, and 1 continues to have frank leakage.

Thomas et al. (7) retrospectively reviewed the continent catheterizable channel outcomes in their patients between 1998 and 2003. A total of 68 Mitrofanoff procedures were performed, using appendix in $43 \%$ of cases, an ileal Yang-Monti tube in $38 \%$ and continent cutaneous vesicostomy in $19 \%$. Stomal stenosis occurred in 9 patients $(13 \%)$ within 1 to 24 months after surgery. False passages with catheterization occurred in four patients $(6 \%)$ with a mean follow-up of 6,5 months. Of the two patients with stomal stenosis both required surgical revision.

The final overall continence rate was $95.45 \%$, therefore similar to other series. Stomal leakage was found in two patients $(9.09 \%)$ requiring a surgical revision: redo procedure in one patient and outlet channel revision (Monti tube) in the other. Both patients are presently continent. The other case of urethral incontinence was an exstrophy patient who is currently awaiting a second bladder neck procedure.
Persistent leakage and stomal complications, mainly stenosis, are the most commonly reported pitfalls of continent urinary diversion. Previous studies have examined the relationship between stomal complications and type of conduit, type of reservoir, and type and site of stoma. Stomal stenosis appears to be independent from all of these variables (10).

It is well known that bladder augmentation and catheterizable urinary diversion lead to an increased risk of urolithiasis. Reasons for this include urinary stasis, mucus production, and chronic bacteriuria. Our incidence of lithiasis was $13.63 \%$, lower than other series. At our institution, all parents are instructed to promote aggressive irrigation of their children's reservoir at least once every 24 hours with 100 to $150 \mathrm{~mL}$ of water. A nurse specialized in pediatric urology and stomal care, monitors our patients regularly to make sure that parents follow established guidelines.

Metcalfe et al. (11) published a series of 500 bladder augmentations in children, revealing bladder stones formed in 20 patients, $1 \%$ with a continent catheterizable channel compared to 10 , and $3 \%$ without a channel. The increased risk of bladder calculi associated with continent channels is in agreement with the study of Brough et al. (12) which is probably due to incomplete emptying of the reservoir based on nondependent catheterization. No difference in prevalence of stone formation as regards ileal or sigmoid segments was reported.

Malignancy and enterocystoplasty is also a growing concern and reports from pediatric bladder augmentation are increasing in frequency. It is believed that the incidence of malignancy will increase with longer follow-up. Pathogenesis likely involves nitrosamines produced by bacteria in urine (13-15). Until the pathogenesis and the etiology are better understood, the mainstay of prevention is regular endoscopic surveillance. In the hope of preventing this disturbing event, some authors have become more aggressive in managing recurrent urinary tract infections and advocate annual surveillance cystoscopy to begin at least 8 to 10 years after augmentation. The role of screening urinary cytology has not been explored but it has merit and warrants further study (11).

We recognize that our mean follow-up of 40 months is not long enough to speculate on long-term 
complications like malignancy or nutritional deficits but this was not the scope of this article. On the other hand, we believe that once a precise indication for augmentation is established, it would make no difference in operating the child after 5 years of within 3.6 years (mean age at present study). It is important to stress that we are not advocating earlier surgery than necessary but only reporting on clinical data retrospectively. We admit, however, that this issue is controversial and many authors would argue that they would leave patients with incontinent stomas or ureterostomies until later on. We respect this argument but we regularly offer patients both options and they in fact help us to decide which procedure to follow.

A second potential criticism on this paper would be that early augmentation may increase work for the family simply to achieve a goal (urinary continence) that other children of their age have not yet reached. However, most of our data comprises patients with myelomeningocele, posterior urethral valves and bladder exstrophy. As we are aware, most of these patients are already undergoing CIC for preventing upper urinary tract deterioration, so that excluding our 4 patients with exstrophy, bladder reconstruction would not add much work in terms of care, quite the contrary if we think that a abdominal stoma is much easier to handle than performing urethral catheterization in wheel-chair bounded children or boys with urethral sensitivity.

We would like to make clear that the scope of this retrospective study was to look back in our bladder augmentation database and evaluate if the rate of complications was higher in this period of life. We are not advocating early surgery but this series comprises a very complex population and most PUV and myelomeningocele patients had upper urinary tract deterioration and recurrent urinary tract infection and required treatment.

\section{CONCLUSION}

Our experience shows that continent catheterizable urinary diversion performed by Macedo's technique is a safe procedure even in small patients below five years, guaranteeing renal function preservation, improvement in quality of life with an acceptable rate of complications. It is important that these patients and their parents have to be included in a nurse-assisted program of clean intermittent catheterization, and be stimulated to promote an adequate emptying of their reservoirs, diminishing the risks of bacteriuria, mucus production, lithiasis and stoma stenosis. Long-term results for children operated on under the age of 5 are still lacking but we do not expect much difference compared to that which is currently reported in the literature.

\section{CONFLICT OF INTEREST}

None declared.

\section{REFERENCES}

1. Gilbert SM, Hensle TW: Metabolic consequences and long-term complications of enterocystoplasty in children: a review. J Urol. 2005; 173: 1080-6.

2. Feng AH, Kaar S, Elder JS: Influence of enterocystoplasty on linear growth in children with exstrophy. J Urol. 2002; 167: 2552-5; discussion 2555.

3. Macedo A Jr, Srougi M: A continent catheterizable ileum-based reservoir. BJU Int. 2000; 85: 160-2.

4. Freitas RG, Nobre YT, Macedo A Jr, Demarchi GT, Ortiz V, Srougi M: Continent urinary reconstruction in rhabdomyosarcoma: a new approach. J Pediatr Surg. 2004; 39: 1333-7.

5. Calado AA, Macedo A Jr, Barroso U Jr, Netto JM, Liguori R, Hachul M, et al.: The Macedo-Malone antegrade continence enema procedure: early experience. J Urol. 2005; 173: 1340-4.

6. Macedo A Jr, Garrone G, Leslie B, Liguori R, Ottoni SL, Ortiz V: A new method to augment the bladder, provide a catheterizable stoma and reconstruct the bladder neck in one operation from a single intestinal segment: The technique step-by-step. J Ped Urol 2006; 2: 84. Abstract no 17.

7. Thomas JC, Dietrich MS, Trusler L, DeMarco RT, Pope JC 4th, Brock JW 3rd, et al.: Continent catheterizable channels and the timing of their complications. J Urol. 2006; 176: 1816-20; discussion 1820.

8. Leslie JA, Dussinger AM, Meldrum KK: Creation of continence mechanisms (Mitrofanoff) without appendix: the Monti and spiral Monti procedures. Urol Oncol. 2007; 25: 148-53. 
9. Vilela ML, Furtado GS, Koh I, Poli-Figueiredo LF, Ortiz V, Srougi M, et al.: What is important for continent catheterizable stomas: angulations or extension? Int Braz J Urol. 2007; 33: 254-61; discussion 261-3.

10. Rapoport D, Secord S, MacNeily AE: The challenge of pediatric continent urinary diversion. J Pediatr Surg. 2006; 41: 1113-7.

11. Metcalfe PD, Cain MP, Kaefer M, Gilley DA, Meldrum KK, Misseri R, et al.: What is the need for additional bladder surgery after bladder augmentation in childhood? J Urol. 2006; 176: 1801-5; discussion 1805.

12. Brough RJ, O’Flynn KJ, Fishwick J, Gough DC: Bladder washout and stone formation in paediatric enterocystoplasty. Eur Urol. 1998; 33: 500-2.
13. Soergel TM, Cain MP, Misseri R, Gardner TA, Koch MO, Rink RC: Transitional cell carcinoma of the bladder following augmentation cystoplasty for the neuropathic bladder. J Urol. 2004; 172: 1649-51; discussion 1651-2.

14. Lane T, Shah J: Carcinoma following augmentation ileocystoplasty. Urol Int. 2000; 64: 31-2.

15. Filmer RB, Spencer JR: Malignancies in bladder augmentations and intestinal conduits. J Urol. 1990; 143 :671-8.

Correspondence address:

Dr. Antonio Macedo Jr.

Rua Maestro Cardim, 560 / 215

São Paulo, SP, 01323-000, Brazil

Fax: +55 113287-3954

E-mail: macedo.dcir@epm.br

\section{EDITORIAL COMMENT}

This retrospective series of children under age 5 years undergoing continent urinary diversion provides food for thought to readers. The short and long term complication rates, including stone formation, ongoing leakage, stomal stenosis and the need for further surgery are similar to those previously published from other centers regarding older patients. The surgical technique described is another novel variation of the Mitrofanoff principle. Clearly, the technical feasibility of performing this surgery in young patients has been established by the authors, but as they rightly point out, the necessity for such surgery at a young age remains debatable. The one death in their series, although perhaps a statistical aberration, speaks to the gravity of performing these procedures in young patients with often compromised reserves. Caution should be advised before considering continent urinary diversion as the standard approach to the young incontinent patient. These patients and their families are often well served with incontinent stomas, and pull-ups or pads until size, maturity and socialization mandate a surgical solution.

\footnotetext{
Dr. Andrew E. MacNeily $B C$ Children's Hospital Vancouver, British Columbia Canada E-mail:amacneily@cw.bc.ca
} 